\title{
El ensayo inédito sobre la vacuna de Ignacio María Ruiz de Luzuriaga (1763-1822)
}

\author{
José Tuells $(*)$ \\ (*) orcid.org/0000-0003-1159-429X. Cátedra Balmis de Vacunología. Universidad de Alicante. \\ tuells@ua.es
}

Dynamis

[0211-9536] 2015; 35 (2): 459-480

http://dx.doi.org/10.4321/S0211-95362015000200009
Fecha de recepción: 9 de enero de 2014

Fecha de aceptación: 24 de enero de 2015

SUMARIO: 1-Introducción. 2.-Composición de los «Papeles sobre la vacuna». 3.- Nota biográfica sobre Ruiz de Luzuriaga. 4.-Los informes de Luzuriaga. 4.1.-El enigma de D. Luis. 4.2.-El informe imparcial. 4.3.-El ensayo sobre la vacuna. 5.-Conclusiones. 6.-Fragmentos del ensayo sobre la vacuna.

RESUMEN: El contenido del conjunto documental conocido como «Papeles sobre la vacuna» archivado en la Real Academia Nacional de Medicina y generado por Ignacio María Ruiz de Luzuriaga (1763-1822) no ha sido estudiado hasta la fecha de forma exhaustiva. Como parte de una amplia investigación sobre estos manuscritos se describe el hallazgo de un texto inédito producido en 1801 por Ruiz de Luzuriaga que, con forma de ensayo, pretendía recopilar los conocimientos sobre el método vacunal sugerido por Edward Jenner durante los inicios de su introducción en España. Su objetivo era establecer un corpus científico y académico sobre la vacuna que facilitara su comprensión, asimilación y buena práctica entre los vacunadores españoles. El texto, contenido en el volumen 3 de los «Papeles», fue consecutivo a otros dos inmediatamente anteriores, la «Carta a D. Luis» y el «Informe imparcial sobre la vacuna». Este estudio analiza el origen y destinatarios de los tres textos, revelando la identidad de «D. Luis» y describiendo los contenidos del «Ensayo», documento hasta ahora desconocido y en el que destaca por su valor historiográfico la primera traducción al español del «Inquiry» de Jenner, realizada por Ruiz de Luzuriaga.

PALABRAS CLAVE: vacuna, viruela, siglo XIX, Ignacio María Ruiz de Luzuriaga, Edward Jenner.

KEY WORDS: vaccine, smallpox, XIXth century, Ignacio María Ruiz de Luzuriaga, Edward Jenner. 


\section{Introducción $(*)$}

Durante la Navidad de 1875, Luis de Foxá dedicó una parte de su tiempo a revisar la biblioteca que había heredado de su padre, fallecido unos meses atrás. Tras consultar a un amigo, el académico Ramón Llorente, tomó la decisión de ceder a la Academia «una pequeña parte de la biblioteca que fue del Doctor D. Ignacio María Ruiz de Luzuriaga». La entrega iba acompañada de una carta dirigida al Presidente de la Academia ${ }^{1}$.

En la Sesión de Gobierno de la Academia del 3 de enero de 1876, Llorente comunicó el contenido de la carta de Foxá, tras lo que se acordó contestar a éste en agradecimiento «al alto aprecio con que la Academia recibe tan generoso e importante donativo» y asimismo nombrar una comisión «que examinara los manuscritos procedentes del Sr. Ruiz de Luzuriaga para entresacar lo que tenga más notable y publicarlo si fuera conveniente» ${ }^{2}$. La comisión la compusieron los académicos Méndez Álvaro, Colmeiro, Iglesias, Pereda y Santucho, y no hay constancia de que se produjera informe alguno.

Luis de Foxá y Muñoz, abogado, era hijo de Luis de Foxá y Garma, Brigadier de Infantería, Caballero Gran Cruz de la Real y distinguida Orden de San Hermenegildo y de Fernanda Muñoz Ruiz de Luzuriaga, fallecidos ambos con seis días de diferencia en enero de $1875^{3}$. Fernanda era hija de Francisco y de María Josefa, ésta última, hermana y una de las herederas de Ignacio María Ruiz de Luzuriaga ${ }^{4}$, hecho que explica el origen y la pertenencia hasta entonces en el seno familiar de los «Papeles sobre la vacuna».

$\left(^{*}\right)$ Este trabajo se inscribe en el marco del proyecto de investigación «Los papeles sobre la vacuna» que ha recibido financiación de la Fundación Española de Vacunología (FEV1-11PA).

1. Carta de Luis de Foxá al presidente de la Academia de 29 diciembre 1876. Real Academia Nacional de Medicina (en adelante RANM) Doc. 5921; El escrito «con firma ilegible» es citado por: Granjel, Luis. Ruiz de Luzuriaga y la Real Academia Nacional de Medicina. Azkoitia: Instituto Internacional Xavier María de Munibe de Estudios del siglo XVIII; 2008. El bibliotecarioarchivero de la Academia, Manuel Iglesias, dio cuenta de la entrada de estos documentos en una nota incluida en la Sesión Inaugural de 1786. Libro de Actas año 1871-1880 RANM, Junta ordinaria celebrada el 3 de enero de 1876.

2. Junta ordinaria 3 de enero 1876, n. 1.

3. La carrera militar de Luis de Foxá y Garma puede seguirse en Estado Militar de España, el fallecimiento fue recogido en Correspondencia de España y Mercurio de España. Las actas de defunción del matrimonio se encuentran en el Libro de Defunciones de 1875 de la parroquia de San Sebastián de Madrid (pp. 305-305v, 308).

4. Testamento de Ignacio María Ruiz de Luzuriaga redactado ante el notario Antonio Moretón. 20 de abril de 1822. Archivo Histórico de Protocolos, Madrid; Signatura 23764; 120-121v. 
La figura de Ruiz de Luzuriaga ha sido estudiada con anterioridad por varios autores entre los que cabe destacar el apasionado panegírico que le dedicó Fabra (1822), la perspectiva familiar trazada por Usandizaga (1964), la nota histórica de López Piñero (1983) o el tributo de Granjel (2008) a su relación con la Academia ${ }^{5}$. Sus trabajos relacionados con la vacuna y contenidos en los «Papeles» también han sido abordados aunque de forma parcial $^{6}$. Nuestro objetivo es dar a conocer un texto inédito y hasta ahora nunca mencionado en la historiografía de Luzuriaga que constituye su intento de producir una obra que compilase los conocimientos y avances sobre la vacunación. Para ello utilizó fuentes bibliográficas nacionales e internacionales así como sus propias experiencias como vacunador y la de otros vacunadores españoles con los que mantuvo correspondencia ${ }^{7}$.

Se analiza a continuación la composición de los «Papeles sobre la vacuna», dedicando a los contenidos de su volumen 3 la mayor atención. En él se encuentran tanto la «Carta a D. Luis», como el «Informe imparcial», ya conocidos, así como el «Ensayo», el documento más extenso de los tres, en el que Ruiz de Luzuriaga incluyó una traducción de la obra de Edward Jenner. El conjunto de esta investigación, con las transcripciones tanto

5. Fabra Soldevila, Francisco. Elogio histórico del doctor en medicina Don Ignacio María Ruiz de Luzuriaga, compuesto y leído en la Academia de Medicina y Ciencias naturales de Madrid el día 19 de noviembre. Madrid: Imprenta de D. León Amarita; 1822; Usandizaga Soraluce, Manuel. Los Ruiz de Luzuriaga. Eminentes médicos vascos ilustrados. Salamanca: Universidad de Salamanca; 1964; López Piñero, José Ma. Diccionario histórico de la ciencia moderna en España Volumen II (M-Z). Barcelona: Ed. Península, 1983; Blasco Martínez, Luis. Higiene y sanidad en España al final del antiguo régimen. Universidad Complutense de Madrid; 1991; Granjel, n. 1.

6. Algunos trabajos sobre la introducción, difusión y propagación de la vacuna en España que han utilizado como fuente documental los «Papeles sobre la vacuna» son: Romeu de Armas, Antonio. La inoculación y la vacunación antivariólica en España: (datos para la historia de la medicina española en los siglos XVIII y XIX). Medicina Española. 1940; 4: 317-329; López Gómez, José Manuel. Contribución al conocimiento de los inicios de la vacunación antivariólica en Cataluña. Gimbernat. 1989; 12: 167-178; Riera Blanco, Manuel; Rigau Pérez, José G. Los primitivos de la vacuna en Tarragona y el ingeniero de marina Don Juan Smith. Gimbernat. 1992; 17: 289-303; Olagüe de Ros, Guillermo; Astrain Gallart, Mikel. Una carta inédita de Ignacio María Ruiz de Luzuriaga (1763-1822) sobre la difusión de la vacuna en España. Dynamis. 1994; 14: 305-337; Olagüe de Ros, Guillermo; Astrain Gallart, Mikel. ¡Salvad a los niños!: Los primeros pasos de la vacuna antivariólica en España (1799-1805). Asclepio. 2004; 56-1: 7-31; Usandizaga Soraluce, n. 5; Blasco Martínez, n. 5.

7. El análisis pormenorizado de la correspondencia contenida en los «Papeles» será objeto de un estudio aparte. 
de las cartas como de los informes y el ensayo de Ruiz de Luzuriaga, está siendo redactado para su edición en formato de libro.

\section{Composición de los «Papeles sobre la vacuna»}

Francisco Fabra Soldevila, académico, íntimo amigo y testamentario, no hizo alusión en su Elogio a los «Papeles» ni al interés de Ruiz de Luzuriaga hacia la vacunación. Sí incluyó una nota sobre su padre, José Santiago Ruiz de Luzuriaga, y el conde de Peñaflorida como introductores de la inoculación de viruelas en el País Vasco. Así mismo, en la descripción de su vasta biblioteca, realizó una cita al manuscrito «Informe imparcial sobre la vacuna, preservativo de las viruelas, descubierto por el doctor Eduardo Jenner» ${ }^{8}$.

En las actas de la Academia correspondientes al periodo 1877-1933 no hay referencia documental a este material. En 1934, año en que la Real Academia Nacional de Medicina (RANM) conmemoraba el bicentenario de la Academia Médica Matritense, se publicó un catálogo de sus obras donde se cita con el número 63 y 64 los «Papeles sobre la vacuna. 2 volúmenes. Manuscrito. 21x15; cartón» ${ }^{9}$.

Se encuentra aquí lo que Rumeu de Armas cita como «correspondencia encuadernada sobre la vacuna de Ruiz de Luzuriaga» y que él mismo fue el primero en relacionar y estudiar, anotando la signatura $\left(8-6^{a}\right)(19-20)^{10}$. Rumeu señala que Luzuriaga «dio por fin a la estampa, en el año de 1801, el fruto de sus observaciones y estudios en un modesto Informe imparcial (...)», documento que estudia de forma separada. El manuscrito nunca llegó a la imprenta. La duda sobre su posible publicación la mantienen, no obstante, Usandizaga ${ }^{11}$ y Blasco ${ }^{12}$. Usandizaga no da más signatura que «Papeles sobre la vacuna. Tres tomos con manuscritos. Biblioteca de la RAM»y, sobre el «Informe imparcial» «Manuscrito, Biblioteca de la RANM» ${ }^{13}$. Blasco reseña por una parte los «Papeles sobre la vacuna (1801-1802), dos manuscritos,

\footnotetext{
8. Fabra Soldevila, n. 5, p. 7 y 52.

9. Academia Nacional de Medicina (1784-1934), Exposición de libros y documentos, Catálogo. Madrid: Impr. de J. Cosano; 1934, p. 43-44.

10. Romeu de Armas, n. 6, p. 321.

11. Usandizaga Soraluce, n. 5, p. 78.

12. Blasco Martínez, n. 5, p. 202.

13. Usandizaga Soraluce, n. 5.
} 
Biblioteca RANM»y, por otra el «Informe imparcial sobre el preservativo de las viruelas, Biblioteca RANM, manuscritos, carp 12, 703» ${ }^{14}$.

Olagüe y Astrain describen los «Papeles sobre la vacuna» como «Signatura 17-2 ${ }^{\circ}$. S. Gobierno 18» y «Signatura 17-2. S. Gobierno 19», mientras que el «Informe imparcial» es citado como «RANM, Papeles sobre la vacuna, 1802. $234^{\text {a }}$ Biblioteca, fols. 1-48v», del que afirman taxativamente «nunca fue editado hasta la fecha» ${ }^{15}$. Por su parte, Granjel cita los «Papeles de la vacuna» como «Archivo de la academia, documento no catalogado« y el «Informe imparcial», que denomina «informe sobre el descubrimiento de Jenner», como «Legajo 11, documento $703{ }^{16}$.

Las signaturas y clasificación de documentos en una biblioteca, como es el caso en la RANM, dependen del espacio y/o de las distintas ubicaciones que haya podido tener, estando pues sujeta a cambios, como ha ocurrido con estos documentos.

Para clarificar el actual detalle documental que conforma los «Papeles sobre la vacuna» hay que decir que constan de cuatro volúmenes, cada uno con su propia signatura:

Volúmenes 1 y 2: 17-2a Sala de Gobierno 18 y 17-2a Sala de Gobierno 19. Son dos volúmenes que, reunidos con una portada común titulada «Papeles sobre la vacuna», recogen las cartas remitidas a Ruiz de Luzuriaga por distintos vacunadores, así como reflexiones, transcripciones de artículos publicados en la prensa francesa y resúmenes manuscritos por el médico vasco, fechados en 1801 y 1802. Están compuestos por 641 cuartillas (386 y 255, respectivamente), cuyo tamaño oscila entre 20 y $22 \mathrm{~cm}$., cosidas y pegadas unas a otras por quien las recopiló, presentando una encuadernación rústica muy liviana que tuvo que ser deshecha al procederse, en los años noventa, a su microfilmación con objeto de salvaguardar los documentos. Fue cuando se numeraron las hojas en su ángulo inferior derecho y se encargó un estuche, simulando su lomo nervios y dorados, donde se conservan desde entonces.

Volumen 3: 23-4ª Biblioteca 14. Con el título «Papeles sobre la vacuna. $1802 »$, este tomo de tamaño folio $(32 \mathrm{~cm}$.) y encuadernación holandesa con tapas de papel jaspeado, consta de una gran variedad de material referente a la vacunación. Del mismo, cabe destacar una versión del «Informe imparcial

\footnotetext{
14. Blasco Martínez, n. 5.

15. Olagüe de Ros; Astrain Gallart, 1994, n. 6, p. 306

16. Granjel, n. 1, p. 29
} 
sobre la preservación de las viruelas...», así como tablas de vacunados, traducciones, proyectos de Luzuriaga. En él se encuentran los documentos sujeto de este estudio.

Volumen 4: Legajo 11, Documento 703. Se trata del «Informe imparcial sobre el preservativo de las viruelas descubierto por el Dr. Eduardo Jenner...», manuscrito con una caligrafía especialmente cuidada, en 97 folios $(32 \mathrm{~cm})$ cosidos y en rústica. Este documento es la versión preparada para remitir a la imprenta.

\section{Nota biográfica sobre Ruiz de Luzuriaga}

La biografía de Ignacio María Ruiz de Luzuriaga (1763-1822) ha sido bien estudiada por la historiografía española ${ }^{17}$. Fue determinante para su formación intelectual el ingreso a los 15 años (1777) en el Seminario Patriótico Bascongado de Vergara en régimen de internado. Aquí se fraguó el interés de Luzuriaga por las aplicaciones de la química a la ciencia médica ${ }^{18}$. Completó su formación de medicina y química en París entre 1780 y 1784, asistiendo a las lecciones impartidas por Fourcroy, D'Arcet, Portal, Sabatier, Jussieu y Dessault. Allí estableció amistad con Philippe Pinel $(1755-1826)^{19}$. Luzuriaga amplió sus estudios en el Reino Unido entre 1784 y 1787, pasando de Londres a Edimburgo, donde tras estudiar con Gregory, Black, Home y Duncan, recibió los grados de Maestro en Artes y de Bachiller y Doctor en Medicina.

Obtuvo entonces varios nombramientos, como corresponsal del Jardín Botánico de Madrid a cargo de su director Casimiro Gómez Ortega que ocupaba, además, el puesto de Académico secretario para las correspondencias extranjeras de la Real Academia de Medicina, como socio honorario de la Sociedad Médica de Londres, miembro del Liceo Médico de la misma ciudad y asimismo, de la Real Sociedad de Medicina y de la Real Sociedad de Historia Natural, ambas de Edimburgo.

\footnotetext{
17. Fabra Soldevila, n. 5; Usandizaga Soraluce, n. 5.

18. Usandizaga Soraluce, n. 5, p. 52.

19. Pinel, Philippe. Compendio de la nosografía filosófica del Dr. Pinel, escrito en latín con arreglo a la última edición por J. S. CH., revisado y aprobado por el mismo Pinel, traducido al castellano por D. Pedro Suarez Pantigo. Madrid: Impr. de D. Miguel de Burgos; 1829.
} 
A su regreso, renunció a la Cátedra de Ciencias Naturales que le había propuesto el Seminario de Vergara y se trasladó a Madrid para ejercer la medicina. Francisco Martínez Sobral, médico de Cámara, ejerció como tutor suyo en la capital y presidió la reválida de los títulos del joven Luzuriaga, de acuerdo con las exigencias del Protomedicato.

El 8 de abril de 1790, a punto de cumplir los 27 años, ocupó una plaza en la Real Academia de Medicina, la institución que sería el centro neurálgico de su actividad científica y donde desplegó una intensa actividad, ocupando sucesivamente los cargos de Secretario, Secretario de Correspondencias Extranjeras y Vice-Presidente (1798 hasta 1807). Pasó en Madrid el resto de su vida.

Luzuriaga ha sido descrito como un trabajador incansable, capaz de interesarse por una gran variedad de temas. Ese rasgo se observa en la cantidad de manuscritos que no vieron la luz por falta de tiempo para materializarlos o simplemente por pasarse a otro sujeto de estudio. Soltero, sin compromisos familiares, retraído y concentrado, su pasión era el estudio, la escritura, la traducción y el acopio de documentos para futuros textos. Según Fabra, Luzuriaga «decía con franqueza que varios de sus manuscritos necesitaban algún retoque y manifestaba también la intención que tenía de revisar sus trabajos para formar una colección útil y ordenada» 20 .

Los libros, su biblioteca, eran la representación más visible de sus entusiasmos. Conservaba tal volumen de libros que tuvo un pleito de desahucio en 1795 por no querer desalojar un cuarto que tenía en la calle Baño $\mathrm{n}^{\circ} 8$, llegando incluso a solicitar la mediación de la corte, «ya por último convencido Luzuriaga de su ningún derecho en justicia, recurrió a la Real Persona, sin duda para alcanzar gracia» ${ }^{21}$, que no fue atendida.

El material bibliográfico que tanto apreciaba quedó reflejado en sus últimas voluntades, sus «bienes consisten la mayor parte en librería, pintura, muebles, alhajas» ${ }^{22}$. Su testamento, hallado recientemente, fue redactado en Madrid el 20 de abril de $1822^{23}$. Fabra fue uno de sus albaceas, otorgándole la potestad de «que hecho cadáver se adorne con el hábito que elijan, a

\footnotetext{
20. Fabra Soldevila, n. 5 .

21. Pleito de Bernardo Sancho Larrea contra Ignacio María Ruiz de Luzuriaga. Archivo Histórico Nacional de Madrid. Consejos 31158, Exp. 11

22. Testamento, n. 4.

23. Tuells, José; Duro Torrijos, José Luis; Díaz-Delgado Peñas, Ignacio. Anotaciones a la biografía de Ignacio María Ruiz de Luzuriaga (1763-1822), el inicio de la vacunación contra la viruela en España. Vacunas. 2012: 13; 128-132.
} 
cuya disposición queda la caja ataúd, funeral, y sitio donde se le sepulte». Dos días después falleció, oficiándose su funeral en la Parroquia de San Sebastián de Madrid. Fue enterrado en un nicho del cementerio de la Puerta de Fuencarral de Madrid ${ }^{24}$.

Luzuriaga instituyó como «únicos y universales herederos por iguales partes, a sus seis hermanos María de Jesús, María Josefa, María Ignacia, Margarita, Ana María Pascasia, y José Manuel Ruiz de Luzuriaga» ${ }^{25}$. Éstos pleitearían más tarde por la herencia. María Josefa fue la depositaria final de, al menos, los «Papeles sobre la vacuna».

\section{Los informes de Luzuriaga}

\subsection{El enigma de D. Luis}

El conocimiento sobre la inoculación de viruelas le llegó a Luzuriaga a través de su padre que, tras practicarla en un hijo del Conde de Peñaflorida el 14 de mayo de 1771, la extendió a otros ocho muchachos de Berriatua ${ }^{26}$. La Sociedad de Amigos del País alentó la variolización en Vascongadas de lo que dio cuenta Timotheo O'Scanlan, el promotor más activo de la inoculación en España, en su obra de 1784 donde publicó la carta de José Ruiz de Luzuriaga que le narraba estos hechos ${ }^{27}$.

Años después Luzuriaga, ya como Secretario de la Academia, se mostraría favorable a esta técnica tal como recogen las actas de la RANM de los años 1796 y 1799. La vieja relación entre Luzuriaga padre y O'Scanlan fue continuada por su hijo que fue compañero de O'Scanlan en la Academia hasta el fallecimiento de éste en $1795^{28}$. La familiaridad con la práctica inoculatoria de Luzuriaga fue avalada por su padre en 1791, que mencionaba a un amigo que «puede hacerlo por medio de un hijo que tengo en Madrid...

24. Certificado de defunción de Ignacio María Ruiz de Luzuriaga, 22 de abril de 1822. Parroquia de San Sebastián, Madrid; Libro defunciones 1822, fol. 73.

25. Testamento, n. 4.

26. Usandizaga Soraluce, n. 5.

27. O’Scanlan, Timotheo. Práctica moderna de la inoculación. Madrid: Imp. Hilario Santos; 1784.

28. Tuells, José. Sobre la utilidad, seguridad y suavidad de la inoculación (1779). Discurso de Timoteo O'Scanlan (1726-1795) en la Real Academia de Medicina. Vacunas. 2014; 15 (1-2): 63-68. 
que acaba de inocular esta primavera a la familia de ... y otras» ${ }^{29}$. Por otra parte, Luzuriaga tenía una estrecha relación con Francisco Martínez Sobral, primer médico de Cámara, que en 1798 intervino en la inoculación de tres infantes de la Familia Real.

Luzuriaga tuvo pues, por tradición familiar, por haberla practicado y por sus relaciones con otros colegas un excelente conocimiento de la variolización, hecho que pudo orientar su predisposición favorable hacia la vacuna. Todos los autores hasta ahora mencionados, excepto Fabra, coinciden en señalarlo como una figura clave de la difusión de la vacuna en España $^{30}$. La base para estas afirmaciones radican en el material contenido en los «Papeles», que pueden considerarse documentos de dos tipos: cartas e informes. Sin embargo, éstos no han sido estudiados en profundidad hasta la fecha. Blasco relaciona cronológicamente una parte importante aunque no exhaustiva de la correspondencia y Olagüe y Astrain analizan la carta dirigida al «Amigo y Señor D. Luis» ${ }^{31}$. Los autores que han abordado el tema la mencionan para documentar la «red epistolar» tejida por Luzuriaga compuesta mayoritariamente por nobles, altos funcionarios del estado y el cuerpo de sanitarios, médicos y cirujanos.

La correspondencia contenida en los Papeles ha dado lugar al reconocimiento que Ruiz de Luzuriaga ha recibido por su papel central como «introductor, propagador, divulgador, defensor» de la vacunación en España. Esta afirmación podría, sin embargo, ser matizada introduciendo un enfoque que contemplara el hecho de que probablemente lo fue malgré lui y que le sobrevino por su posición en la Academia, por su reconocido prestigio científico en Madrid y por sus relaciones nacionales y foráneas. Luzuriaga se vio impelido a dinamizar la vacunación por la fuerza de varias circunstancias concurrentes que se desgranan a continuación.

Los documentos que permite identificar los inicios de la vacunación en España podrían, a la luz de un análisis detallado, dar nuevas claves sobre las dinámicas que se establecieron durante aquellos meses de vértigo. Si la correspondencia refleja la malla tejida por Luzuriaga y muestra al colectivo de actores interesados por la vacuna, los informes revelan su intento de plasmar tanto sus experiencias como un compendio de la técnica vacunatoria y su justificación científica. Conviene señalar aquí el papel central de Ignacio

\footnotetext{
29. Usandizaga Soraluce, n. 5, p. 42.

30. Blasco Martínez, n. 5; López Piñero, n. 5; Usandizaga Soraluce, n. 5; Granjel, n. 1

31. Blasco Martínez, n. 5; Olagüe de Ros; Astrain Gallart, 1994, n. 6.
} 
de Jaúregui como estimulador de Luzuriaga para que éste produjese un texto útil y de referencia para los vacunólogos españoles del momento ${ }^{32}$.

El uso del término «informes» no hace referencia a los dos ejemplares del «Informe imparcial» (volúmenes 3 y 4) que se conservan sino a los tres manuscritos que Luzuriaga redactó con distinta intención durante el segundo semestre de 1801, contenidos en el volumen 3 de los «Papeles». El primero es la carta al «Amigo y Sr. Don Luis», el segundo es el «Informe imparcial»y el tercero es el «Ensayo sobre la vacuna». En el transcurso de esos meses, Luzuriaga fue ampliando el formato narrativo de sus experiencias pasándolas de carta a informe y luego a ensayo.

La carta al «Sr. D. Luis» ha sido bien analizada y transcrita por Olagüe y Astrain $^{33}$. Los autores refieren no haber identificado a «Don Luis» y apuntan que podría tratarse de un español vinculado a la Sociedad Bascongada de Amigos del País residente en París, aunque no descartan la hipótesis de que fuera francés y que bien pudiera ser médico. La intuición es acertada.

El «Sr. Don Luis» es Joseph-Louis Proust (1754-1826), químico y farmacéutico francés, miembro de la Academia de Ciencias y de la Academia Real de Medicina de París, científico de gran prestigio al que se debe la enunciación de la ley de proporciones definidas o la identificación del azúcar de las uvas como glucosa. Proust pasó casi treinta años de su vida en España, en una primera estancia (1778-1780) fue profesor de química en el Seminario de Vergara, en una segunda (1784-1806) fue contratado para dirigir la cátedra de química y metalurgia en el Real Colegio de Artillería de Segovia, pasando a Madrid en 1799 para dirigir el Laboratorio Real. En 1806 volvió a Francia y dada la situación política se instaló definitivamente en Craon, localidad próxima a Angers su ciudad natal ${ }^{34}$.

Proust, antiguo profesor de Luzuriaga en Vergara donde entablaron amistad, fue también compañero en la Academia de Medicina madrileña. Luzuriaga lo cita como el «célebre Chîmico D. Luis Proust» en su Disertación sobre el cólico de Madrid (1796) ${ }^{35}$ y ambos publicaron artículos en el Journal de Physique, chimie, d'histoire naturelle et des arts, dirigido inicialmente por

32. Un total de 23 cartas de la correspondencia contenida en los «Papeles» se deben a Jáuregui, cuya influencia en este proceso está siendo analizada en otro texto.

33. Olagüe de Ros; Astrain Gallart, 1994, n. 6.

34. Fournier, Josette. Louis Joseph Proust (1754-1826) était-il pharmacien? Histoire de la pharmacie. 1999; 321: 77-96.

35. Astrain Gallart, Mikel. Tres manuscritos inéditos de Ignacio María Ruiz de Luzuriaga sobre la higiene naval. Dynamis. 1991; 11: 437-463. 
François Rozier (1734-1793) y más tarde por Jean Claude de La-Métherie (1743-1817).

El 23 de junio de 1801, la Comisión de la Vacuna de la Sociedad de Medicina de París con sede en el Louvre remitió una carta a la Academia en la que solicitaba les informaran sobre el estado de la vacuna en España y les contestaran a unas preguntas sobre la confianza que ésta merecía a la población, si existían detractores, desde cuando se vacunaba y desde qué país se había recibido la vacuna. Adjuntaban también el primer informe sobre la vacuna que habían realizado y prometían mandar el segundo. Firmaban la carta Emmonot (Secretario), Sedillot (Presidente), Ané y Roussille-Chamseru.

Junto a esta carta, también en los «Papeles», se encuentra otra redactada en parecidos términos, aunque sin firma ni fecha, al final de la cual se revela la conexión entre Proust, La-Métherie y Luzuriaga:

«Laméterie salue Mr Proust et le prie de se souvenir des demandes qui lui ont été faittes par un de ses collègues de la Commission de la Vaccine lesquelles lui ont été transmises para Mad. son épouse et de lui envoyer la réponse le plutôt qu'il lui sera possible, persuadé qu'elles seront plus exactes que celles qu'ont a reçut jusqu'à ce moment» ${ }^{36}$.

En París se habían establecido dos entidades para hacer un seguimiento de los progresos de la vacuna. La primera, creada en febrero de 1800 por el Duque de Larochefoucault-Liancourt como una sociedad de suscriptores, fue denominada Comité Central de la Vaccine, de la que formaron parte Thouret (Presidente), Husson (Secretario), Pinel, Colon, Guillotin, Delaroche, Halle, Tourlet, Roussille-Chamseru, entre otros. Produjeron varios documentos, entre los que se encuentra el Rapport du Comité Central de la Vaccine ${ }^{37}$.

Justo un año después, en febrero de 1801, se creó la Commission de Vaccine séante au Louvre, auspiciada por la Societé de Médecine. La integraron Emmonot, Ané, Sédillot, Bodin, Dessesartz, Moreau, Daval, Brewer, Allan y Roussille-Chamseru (estuvo en las dos), entre otros ${ }^{38}$. Su papel fue

36. Carta de la Comisión de la Vacuna de París a la Real Academia de Medicina de Madrid. RANM, Papeles de la Vacuna, Signatura 17-2a S. gobierno, 18, fols. 3-9

37. Rapport du Comité Central de Vaccine, établi a Paris par la Societé des souscripteurs pour I'examen de cette découverte. Paris: Imprimerie de Guilleminet; 1803.

38. Meynell, Elinor. French reactions to Jenner's discovery of smallpox vaccination: The primary sources. Social History of Medicine. 1995; 8: 285-303. 
menos importante, solo produjeron dos Rapport $(1801,1802)$ con los datos recabados de una serie de informantes. Su primer Rapport fue remitido a la Academia como ellos citan en su carta y fue rápidamente traducido al español por Manuel de Ascargorta, secretario del Duque de Osuna, como informa el propio Luzuriaga en su carta a «Don Luis» ${ }^{39}$.

La Commission du Louvre escribió a varios referentes españoles, alguno de los cuales contestó a la invitación como fue el caso de Francesc Salvá i Campillo (lo citan en el Rapport de 1802). Luzuriaga redactó la carta a «D. Luis», esto es, a Proust, con la idea de que la hiciera llegar a la comisión francesa. No hay constancia de que llegara a su destino. En ella hacía referencia al texto ya traducido por Ascargorta, reconocía que Piguillem, Salvá y Mitjavila fueron los iniciadores de la vacunación en España y destacaba el papel de Jáuregui, Onís y Castillo en la introducción de la vacuna en la corte $^{40}$, citaba algún caso de efectos adversos y enumeraba un listado de vacunadores y del número de vacunados. Relataba expresamente que se trataba de un «borrador de la propagación de la vacuna en España» y repetía que «basta este borrador mientras comunico en mi obra el resultado...». Finalmente concluía «no me comprometan Vds., en el extracto que hagan entre Vmd., y el Dr. Pinel, a quien saludo». A la relación de amistad con Proust, hay que añadir la que también mantuvo con Pinel, que lo cita en su Nosographie Philosophique. Los tres habían publicado en el Journal de Physique, que tenía por aquella época problemas con la censura española, de ahí sus precauciones.

39. El propio Luzuriaga recoge esta mismo información en una carta dirigida a Luis Onís en la que indicaba: «Dn Manuel de Ascargorta, hijo del Secretario del Duque de Osuna, acaba de publicar la traducción del primer informe dirigido a la Sociedad de Medicina de París, por la Comisión Médica establecida en el Louvre». RANM, Papeles sobre la vacuna 1802, signatura 23-4a Biblioteca 14, fols 326-345.

40. «Los médicos de Cataluña son los primeros que han tenido la gloria de introducir la vacuna en aquel principado [...]. Por el mes de Abril, el Sr. Castillo, Secretario de embajada en París, [...] remitió entre cristales el fluido vacuno, con el cual fue inoculada en Aranjuez María Conrote, edad de 9 años [...]. Con la materia de esta se inocularon a los 9 días de la vacunación dos hermanas de la vacunada, y con la de estas últimas, la hija del Sor. Onis, de la Secretaría de Estado, de 4 años de edad, y la del Sor. Campuzano, de la Secretaría de Marina, de 18 meses, practicándose todas estas vacunaciones por Dn. Tomás Bueno, Cirujano, bajo la dirección del Dr. Dn. Ignacio Jáuregui, Médico de la Real Familia, y uno de los más ardientes promotores de la vacuna, quien continúa propagándola en los Reales Sitios con la mayor felicidad; de manera que participa juntamente con los Sres. Castillo y Onís de la gloria de haberla introducido en la Corte». RANM, Papeles sobre la vacuna, n. 39. 
Luzuriaga pedía en la carta los originales de Jenner ya que no disponía ni siquiera de la traducción al latín que hizo Aloisio Careno ${ }^{41}$. Finalmente en las alusiones personales se refería a conocidos de la época de Vergara, como Félix Samaniego, Valentín de Foronda, Mariano Luis de Urquijo, Eugenio Izquierdo - un personaje clave ya que conoció a Proust en París y fue el que lo recomendó al Seminario de Vergara $-{ }^{42}$, para acabar dándole recuerdos de Mari Pepa, que no era otra que su propia hermana María Josefa Luzuriaga.

Este primer «borrador» en forma de carta es, pues, un avance de la situación de la vacuna que Luzuriaga elaboró para que Proust y Pinel, sus amigos, difundieran en Francia los resultados españoles, bien en el Journal de Physique bien entregándolo a la Comisión de la Vacuna.

\subsection{El «Informe imparcial»}

Tampoco vio la luz el segundo informe, esto es el «Informe imparcial», más conocido y citado, transcrito en la tesis inédita de Blasco ${ }^{43}$. La redacción de este informe fue generada por la presión del Protomedicato, deseoso de dar respuesta pública al estado de opinión que se estaba formando por la difusión de los sucesos adversos ocurridos tras las vacunaciones practicadas entre los meses de julio y septiembre de $1801^{44}$. Manuel Gorgullo, secretario de dicha institución, le franqueó en octubre un escrito indicándole que:

«por decreto de 16 del corriente ha acordado el Real Protomedicato se comunique a $\mathrm{Vm}$. el presente a fin de que le informe lo que haya observado sobre los sucesos prósperos y adversos de la vacunación, [...], lo que espera ejecutará Vm. con la posible brevedad» ${ }^{45}$.

41. «Deseo las obras originales de Jenner, Woodville \&, y si no la traducción latina del primero publicada en Viena». RANM, Papeles sobre la vacuna, n. 39.

42. "Se murió en La Guardia Dn. Félix Ma Samaniego, nuestro común amigo, de una fiebre pútrida. Urquijo está con la unción en el Castillo de Pamplona desahuciado de los Médicos. Izquierdo está haciendo la visita de gabinete Inspector, consejero de guerra, $\&$, y me temo que saldrán muchos comprometidos en esta visita [...]. A Foronda han hecho cónsul de los Estados Anglo-Americanos con mil doblones de sueldo». RANM, Papeles sobre la vacuna, n. 39.

43. Blasco Martínez, n. 5.

44. Usandizaga Soraluce, n. 5, p. 78-79.

45. En carta fechada a mediado de octubre de 1801 de Manuel Gorgullo dirigida a Ruiz de Luzuriaga. RANM, Papeles de la vacuna, Signatura 17-2a S. gobierno, 18, fol. 11. 
Luzuriaga iniciaba el «Informe imparcial» con una breve introducción latina de Böerhaave y, dirigiéndose «al Sabio Tribunal», proponía que la vacuna debiera denominarse, «deseando perpetuar el nombre de Jenner», como «fluido yennerino» y a su práctica «yennerización» ${ }^{46}$. Volvía a relatar la introducción de la vacuna tanto en la corte como en Madrid, reconociendo la destacada labor del secretario de la embajada en París, Juan del Castillo y Carróz, del Oficial de la Secretaría de Despacho, Luis de Onís y de Ignacio de Jáuregui. Proseguía con la relación de vacunaciones practicadas, deteniéndose en los casos utilizados para desacreditar la vacuna y concluía con un alegato sobre los beneficios que reportaría su generalización.

Luzuriaga enfatizó el número de vacunados, cuya cifra imputada a los de Madrid y la corte (Luzuriaga, Zunzunegui, Azaola, Azcue, Canedo, Lamano, Jáuregui...) resultaba de 826 con una corrección final que daba un total de 860, añadiendo luego que «de sus corresponsales» del resto de España había llegado a sumar 1.126 vacunados. La suma de 860 es el resultado de los 826 de Madrid, más los de Jáuregui en Aranjuez.

Otro documento no incluido en los «Papeles sobre la vacuna» que se encuentra también en la biblioteca de la RANM es un resumen en francés del «Informe imparcial», denominado «Extrait du Rapport manuscrit du Dr. Luzuriaga sur la vaccination ou Jennerisation qui a pour epigraphe le passage suivant de Boerhaave...» ${ }^{47}$. El texto se limita a sostener la idea de Luzuriaga de llamar yennerización a la vacunación y, sobre todo, hace un conteo de los vacunados, cuyo número son idénticos a los del «Informe» (860 y 1126). Este manuscrito fue redactado con la idea de remitirlo a Francia.

Aunque en el análisis de la carta de «D. Luis» se sostiene que los datos son distintos y que existen unas «tablas españolas»y otras «tablas francesas» ${ }^{48}$, se trata de la misma estadística, 860 en el «Informe» y 795 en la «Carta», donde no computa las vacunaciones realizadas por Manuel Andrés, cirujano del marqués de Valmediano, desde el 30 de agosto al 6 de octubre. En los subtotales existen pequeñas diferencias. En la carta a «D. Luis» Luzuriaga se atribuye los vacunados por Azaola (se «yennerizaba» a

46. Informe imparcial sobre el preservativo de las viruelas descubierto por el Dr. Eduardo Jenner, de la Real Sociedad de Ciencias y Artes y de las sociedades médicas de Londres, Médico de Cámara de S.M. Británica etc. Véase: RANM, Papeles sobre la vacuna 1802, 23-4a Biblioteca, fols. 1-48.

47. Tiene dos páginas y media manuscritas y el resto son tablas de vacunados hasta un total de16 páginas. RANM, Carpeta 15, doc. 885.

48. Olagüe de Ros; Astrain Gallart, 1994, n. 6. 
los niños por este cirujano en la librería de la casa de Luzuriaga, mientras éste examinaba a los niños que debían o no ser tratados) lo que provoca una aparente disgresión, pero el resultado es el mismo.

\subsection{El «ensayo sobre la vacuna»}

Lo más novedoso de esta investigación sea quizás el hallazgo de un ensayo de Luzuriaga sobre la vacuna. Se encuentra en el volumen 3 de los «Papeles» y constituye el intento de realizar una auténtica obra de recopilación de todo el conocimiento existente sobre la vacunación ${ }^{49}$. El texto comienza con una dedicatoria «A los niños» y va dirigido a un «Excmo. Sor.» que no es otro que el primer Secretario de Estado y Despacho del Rey, Pedro Cevallos Guerra, lo que subraya el carácter «oficial» que pretendía tener el texto, en formato de libro. Luzuriaga intentaba involucrar a las altas esferas gubernamentales y expresaba en la introducción un contundente mensaje en el que resaltaba los beneficios que la vacunación reportaría al Estado. Una idea compartida por otros ilustrados como, por ejemplo, su compañero en el Seminario de Vergara, Valentín de Foronda, que también se había dirigido a Cevallos manifestando su apoyo incondicional tanto a la inoculación de la viruela como a la vacuna ${ }^{50}$.

Luzuriaga iniciaba el texto un recorrido histórico con numerosas citas bibliográficas sobre obras y textos relativos a la vacuna, así como de los autores más representativos, tanto de la inoculación de viruelas como de los textos publicados después de la obra de Jenner. Proponía desde su perspectiva de salubrista «se renueven contra la viruela los mismos reglamentos que regían con la lepra» para lo que enumeraba un total de 12 puntos. Entre los autores citados se encuentran Böerhaave, Monro, Rose, Le Camus, Rast, Rhazes, Martín de Villanueva, Paulet, Francisco Gil, O'Scanlan, Voltaire, hasta llegar a Jenner.

Tras introducir la figura del cirujano inglés, señalaba «oigamos al mismo doctor Jenner» y a lo largo de quince folios traducía literalmente los 23 casos del Inquiry de Jenner ${ }^{51}$, lo que supone la primera e inédita versión

\footnotetext{
49. RANM, Papeles sobre la vacuna 1802, signatura 23-4a Biblioteca 14, fols 124-264.

50. Foronda, Valentín. Cartas sobre la policía. Madrid: Imp. de Cano; 1801.

51. Jenner, Edward. An Inquiry into Causes and Effects of Variolae Vaccinae, a Disease, discovered in some of the Western Counties of England, particularly Gloucestershire, and Know by the Name of Cow Pox. London: printed for the author by Sampson Low; 1798.
} 
en castellano de esta obra. La traducción es bastante correcta. Sorprende, no obstante, la obstinación de Luzuriaga por denominar «viruela Africana» a lo que Jenner denominaba variolous matter, variolous infection, variolous inoculation o smallpox, utilizando el término «vacuna» o «viruela de las vacas» cuando el inglés empleaba cowpox. Luzuriaga lo justificó en varias ocasiones, argumentando que «en lugar de llamar viruela natural a la variola, es evidente que nos vino de África esta plaga exterminadora de la infancia»o «la viruela Africana, variola de los latinos y que hemos llamado impropiamente viruela natural».

Posteriormente, dedicaba citas a los primeros vacunadores ingleses (Simmons, Pearson, Woodville), para pasar luego a los europeos como Odier y a los miembros de la «Comisión médica para la inoculación de la vacuna», es decir, a los miembros del Comité Central de la Vacuna de París.

Relataba después cómo se había introducido la vacuna en Madrid, justificando la ausencia de contrapruebas debido al poco tiempo que llevaba implantada. Continuaba abordando temas, como el valor de las estadísticas favorables de la vacunación, cómo había de realizarse ésta en los niños, cómo habían dado ejemplo los hombres de Estado y los aristócratas para incentivar la vacunación, los posibles efectos adversos (citando los casos españoles) y cómo había de practicarse la vacunación en embarazadas. Efectuaba una relación extensa de algunos casos de vacunados por él o por Jáuregui, tras lo cual volvía a introducir las «reflexiones que hace el doctor Jenner acerca de las incisiones», nueva traducción de párrafos de la segunda obra de Jenner que también conocía ${ }^{52}$.

A continuación repasaba las obras francesas de Colon, Moreau y Husson, extendiéndose en la reflexiones de estos autores y en los informes del Comité Central de la Vacuna y de la Comisión de la Vacuna del Louvre. Relataba cronológicamente parte de las vacunaciones efectuadas en España hasta el mes de septiembre de 1801, acabando con un apartado que titulaba «resta que expongamos las razones que nos han movido a adoptar este precioso invento de la vacunación ${ }^{53}$, que contenía los siguientes subepígrafes: «La vacunación preserva la viruela Africana» (folios 238-242), «De la viruela vacuna» (folios 243-258), «Desarrollamiento de la vacuna en el hombre»

52. RANM, Papeles sobre la vacuna 1802, n 60, fols. 205-206. Luzuriaga disponía de la segunda obra de Jenner que cita como: Further observations on the variolae vaccinae on Cow Pox, by Edward Jenner, M. D. F. R. S. F. L. S. \& c London, 1799, 4ㅇ, p. 64.

53. RANM, Papeles sobre la vacuna 1802, no 60, fol. 238 
(folios 258-262), «Procedimiento el más seguro que hay para conservar el vacuno y remitirlo lejos» (folios 262), «Observaciones» (folios 262-264) ${ }^{54}$.

Sin duda nos encontramos ante el texto que le había sido reclamado tanto por los funcionarios de la Secretaría de Estado, Onís, Castillo, Pizarro, y fundamentalmente por el propio Jáuregui, que le había escrito desde Aranjuez en agosto de 1801:

«la confusión de folletos que diariamente van saliendo al público y las equivocadas noticias que en ellas se dan acerca de la vacunación se atribuyen a nuestra pereza en dar la disertación que cortaría enteramente el comercio que hacen los autores de aquellos, y el vicio con que el público recibe de ellos las ideas $»^{55}$.

La publicación de la obra de Hernández, la traducción de la obra de François Chaussier, la traducción de Piguillem del texto de Colon, e incluso la noticia de que ya se estaba traduciendo la obra de Moreau (a cargo de Balmis $)^{56}$ hacían que Jáuregui instigase con más fuerza a Luzuriaga para que concluyera el ensayo o disertación. Jáuregui tenía noticia tanto del formato y «láminas a introducir» como de los contenidos, a los que hizo varias correcciones.

Luzuriaga citaba en su ensayo estas iniciales traducciones españolas, refiriéndose a la de Moreau como «esta obra clásica se está imprimiendo actualmente y se publicará con el título de Tratado histórico y práctico de la vacuna, y le dedicará al apreciable y célebre Jenner». También disponía de material bibliográfico inglés (Jenner, Woodville), varios libros y artículos franceses e incluso la traducción latina de Careno, que debió recibir tras redactar la «Carta a D. Luis», en la que los reclamaba. El conjunto del

54. En el apartado final de documentos se incluyen dos fragmentos del texto.

55. Carta de Ignacio Jáuregui a Luzuriaga 31 de agosto de 1801. RANM, Papeles sobre la Vacuna, Signatura 17-2a S. gobierno, 18, fols. 55-56.

56. Hay constancia en el Libro de Actas de la RANM de la asistencia de Balmis a varias sesiones durante los meses de enero a marzo de 1801. Es en ese momento cuando Balmis se incorpora a la fiebre de las traducciones de obras sobre la vacuna eligiendo el texto de Moreau por «tratarse de la más completa y sabia que se ha publicado en la culta Europa y que podría esclarecer en España como conviene la opinión pública e introducir y propagar la benéfica práctica de la inoculación de la vacuna verdadera». Su primer borrador lo remite durante el mes de noviembre, fechas que concuerdan con la afirmación de Luzuriaga que estaba al tanto de esa traducción. Vease Tuells, José. El proceso de revisión a la traducción de Francisco Xavier Balmis del Tratado histórico y práctico de la vacuna, de Moreau de la Sarthe. Gaceta Sanitaria. 2012; 26: 372-375. 
«ensayo» no está pulido, muestra una buena puesta al día bibliográfica, pero carece de vertebración y tiene las características de un primer borrador redactado de forma apresurada.

La obra, impulsada por miembros del aparato del Estado, pretendía ser la referencia española en materia de vacunación. El prestigio de Luzuriaga parecía poder ejecutarla, pero no vio la luz. Luzuriaga se vio desbordado por los acontecimientos, las solicitudes institucionales que le requerían información, la atención a los corresponsales de su «malla» epistolar en los que consumía su afán por divulgar y, a la vez, obtener información, además del propio ejercicio de la vacunación en su domicilio supervisando la labor de cirujanos como Azolea. Hay que añadir que Luzuriaga dedicó en esa época parte de su tiempo a otras cuestiones sanitarias como la fiebre amarilla, lo que podría justificar la falta de conclusión de sus textos.

Aunque participó en el intento de dedicar una sala en las dependencias de la Academia para efectuar vacunaciones gratuitas ${ }^{57}$, su actividad en relación con la vacuna derivó, a partir de 1802, hacia otros temas. Entre ellos, la comprobación de la veracidad de las pretensiones de Hortet o Heydeck ${ }^{58}$ y la emisión de algún dictamen desde la RANM. Finalmente, sus opiniones a favor de la vacuna y ante la observación de su decaimiento en 1804, fueron tomadas en consideración e inspiraron el Real Decreto de Vacunación de $1805^{59}$. Es interesante el documento de diciembre de 1803, elaborado por Luzuriaga como respuesta a un oficio del académico Juan Peñalver, donde apuntaba la necesidad de establecer en la corte y las capitales del reino una vacunación metódica, crear una comisión central al modo de otros países, elaborar instrucciones en forma de catecismos sobre el método de practicar las vacunaciones, vacunar a los niños a los

57. Libro de Actas año 1791-1814 RANM, junta ordinaria celebrada el 8 de octubre de 1801; Minuta de una exposición dirigida al rey referente a la práctica de la inoculación de la vacuna. RANM, Fol., 2, leg. 12C, doc. 60. «El señor Presidente propuso a la Academia que se hiciese una representación a S.M. que se digne permitir y autorizar este cuerpo a imitación de otros cuerpos facultativos de otras Academias de Europa, el practicar la vacunación en la Sala de la calle del Palacio viejo».

58. Olagüe de Ros, Guillermo. De las falsificaciones en la historia: Juan José Heydeck (n. 1755) y su portentoso descubrimiento de una vacuna contra la viruela. Asclepio. 2007; 59 (1): 275-284.

59. En ella se recoge el siguiente texto «Uso y conservación del fluido vacuno en los hospitales de las capitales, bajo las reglas que se expresan». Novísima Recopilación de las Leyes de España. Libro VII. Título XXXVIII. Ley XIX. Real Cédula de 21 de abril de 1805. 
dos meses de edad, etc. ${ }^{60}$. La idea de establecer esa organización estaba contenida en el reglamento de la Expedición de Balmis, de fecha similar, y las Juntas de Vacuna tuvieron cierto éxito en América ${ }^{61}$. Luzuriaga hacía mención muy de pasada a esta empresa de la Corona mostrando cómo la Academia fue ajena al proceso $^{62}$.

\section{Conclusiones}

El texto hallado entre los «Papeles» y denominado por Jáuregui y Luzuriaga como «Ensayo», es un continuo de la «carta a Don Luis» y del «Informe imparcial». Su elaboración responde a peticiones diferentes. La carta es un borrador informativo generado a demanda de las comisiones extranjeras, teniendo por objetivo reflejar en el exterior el estado de la cuestión en España. El informe, realizado bajo la presión del Protomedicato, tiene un valor estadístico, relator de las consecuencias de la vacuna y pretende minimizar las opiniones desfavorables aparecidas tras la ocurrencia de algunos efectos adversos que fueron divulgados por la prensa, pretendiendo pues contrarrestar la presión mediática. Finalmente, el ensayo fue el intento fallido de elaborar por autores españoles un «corpus» sobre el nuevo método que compendiase el «estado de la cuestión vacunal» en lugar de recurrir a traducciones y que sirviese de guía o manual para llevar a cabo la vacunación conforme a reglas de buena práctica.

60. Libro de Actas año 1791-1814 RANM, junta ordinaria celebrada el 9 de diciembre de 1803; Informe de Ignacio María Ruiz de Luzuriaga explicando el descubrimiento, aplicación de la vacuna y demás medidas preventivas. RANM, Fol. 4, leg. 11, doc 698. Peñalver solicitaba que la Academia «piense en los medios de conservar el fluido vacuno y prevenir toda posibilidad de que llegue un día a faltar, exponiendo los medios de propagarla para disminuir los estragos que produce en el Reyno».

61. Tuells, José; Ramírez Martín, Susana María. Francisco Xavier Balmis y las Juntas de Vacuna, un ejemplo pionero para implementar la vacunación. Salud Pública Méx. 2011; 53 (2):172-177

62. Luzuriaga en línea con la idea ilustrada de intervención del Estado en acciones de salud pública como proyecto de biopolítica citaba que «de esta manera sellará el Gobierno el inmenso beneficio que acaba de proporcionar a nuestras Américas con la expedición de la vacuna que será causa de que se pueblen en breve las pampas y desiertos de Buenos Ayres y de que se abaraten los Azúcares, conservando los Negros empleados en sus ingenios que sacrificaba la viruela, como los muchos Indios y Colonos que destruye esta plaga» RANM, n. 60. 


\section{Fragmentos del «ensayo sobre la vacuna»}

A continuación se reproducen dos fragmentos del «ensayo sobre la vacuna»
cuya extensión hace imposible su transcripción completa.

\section{«Dedicatoria e introducción ${ }^{63}$}

\section{A los Niños}

Vuestras caricias, vuestra ternura y delicadeza, expuesta en cuanto nacíais a ser segados por una funesta plaga, así como la lozana flor de mayo se marchita con la escarcha, destruyendo en un momento el fruto de los afanes y esperanzas del jardinero han interesado al Dr. Eduardo Jenner en vuestro amparo, proporcionando un antídoto que os dé una nueva vida puesto que la vuestra, antes de haber pagado el cruel tributo a la viruela se reputaba por muerta y poco segura. Corresponded a tan grande beneficio cuando, libres de esta plaga, lleguéis a ser grandes comentando las artes conservadoras de los hombres, despreciando los mezquinos intereses mercantiles que hacen verter tanta sangre, reíros de los vicios de vuestros predecesor[es], aumentad el uso de sus cortas virtudes y buscad en la Naturaleza misma los medios de ser más felices.

\section{Excmo. Sor.}

Un descubrimiento médico destinado a extirpar la viruela devastadora, a aumentar incalculablemente la población del globo conocido, a evitar las cargas onerosas al Estado, reduciendo el $n^{\circ}$ de ciegos, tuertos, sordos, estúpidos y demás lisiados por la viruela Africana, a conservar la belleza del sexo por cuya falta quedan sin colocar muchas núbiles, a conservar los herederos a las casas que perpetúen el lustre y esplendor de las familias, empequeñeciendo las lágrimas que hasta ahora ha costado esta ponzoña a la ternura paternal, no puede desmerecer la protección de un Ministro ilustrado que tiene en su mano el timón de las nave política, que con la vacunación de su propia hija y sobrino, más que con las órdenes, ha dado un ejemplo al Reyno que no tardarán en imitar todos los que estén sobresaltados por los frecuentes estragos que amenazan de continuo a los que no hayan rendido el tributo que exige este cruel azote de la ${ }^{64}$ infancia.

La sagacidad del Excmo. no puede menos de calcular que si la ${ }^{65}$ Gran Bretaña, a pesar de sus continuas guerras, de las emigraciones repetidas, y las poblaciones de tantas colonias tiene 16 millones y más de población en su recinto menor que el

63. Dedicatoria e introducción al ensayo de la vacuna. RANM. Papeles sobre la vacuna 1802, 23-4a Biblioteca, fols. 124-127.

64. Tachado: humanidad.

65. Tachado: Inglaterra. 
nuestro, a causa de haber generalizado la inoculación de la viruela Africana en todo el siglo pasado, adoptando así como las Naciones civilizadas la vacunación más segura y menos arriesgada, aumentando su población, y sus riquezas harán tributar de su poder a las Naciones que no le adopten y propaguen generalmente.

De qué servirá la propagación de este feliz descubrimiento si las luces políticas de V. E., no facilitan al mismo tiempo la repartición de bienes y herencias en pequeñas porciones, suprimiendo las instituciones góticas de mayorazgos, imposibilitando su fundación, repartiendo por iguales partes las herencias entre todos los hijos, facilitando el que se transmita entre muchos coherederos y fomentado por todos la Agricultura, las artes y las ciencias, la industria y arrancando la propiedad de las manos muertas.

El descubrimiento del inmortal Jenner, combinado con estas miras políticas, hará hacer progresos rápidos a la población, y si la estimación debe ser en razón inversa de su utilidad real, tantos escollos vencidos proporcionarán a V. Excmo., la dulce satisfacción de haber sido útil a su patria y las bendiciones de sus conciudadanos ${ }^{66}$. A la sombra del inmortal Jenner y de la utilidad del descubrimiento, merecerá este ensayo la indulgencia de V. Excmo., cuya vida prospere el cielo muchos años para el bien del Estado».

\section{«Procedimiento para conservar y transportar la vacuna ${ }^{67}$}

El fluido vacuno que se conserva en hilo se seca como un barniz, tiene el grande inconveniente de que forma escamas y no se conserva enteramente en ellas, en cuyo caso no surte regularmente efecto, o produce falsa vacuna. El que se ha conservado en la lanceta cría orín o se oxida el acero y esto lo desmejora y hace mudar de naturaleza, aunque para pocas horas puede trasladarse en ella, de una parte a otra. Aunque no se oxida la lanceta adquiere el fluido vacuno la consistencia de vidrio, diáfana, que corta con sus bordes y que al introducirla en la piel obra como cuerpo extraño, induce una irritación física muy pronta y produce una vacuna bastarda, que no preserva la viruela Africana.

El mejor medio y más conveniente de conservar bien el fluido vacuno, así para practicar la vacunación cuando no hay oportunidad de fluido vacuno reciente como para remitirlo a otras partes comunicando a ellas el beneficio que nosotros hemos tenido, es en vidrio o cristal.

Se toman vidrios cuadrados del tamaño de pulgada y media o dos, en cuadro o circulares. Se tomará uno de estos y se aplicará varias veces sobre la vejiguilla ya abierta, de cuya manera se pega el fluido vacuno al cristal, como se conocerá por el empañado que se distinguirá al trasluz. Se toma el fluido con la punta de la lanceta que se limpia sobre la superficie del cristal para que deponga en ella todo el fluido que se ha cargado en ella, repitiendo varias veces esta operación para cargar bien el cristal,

66. A continuación, tachado: Reciba, pues, V. Ex., con benignidad este ensayo digno.

67. RANM. Papeles sobre la vacuna 1802, 23-4a Biblioteca, fols. 214-216. 
y ambas operaciones en los dos cristales, que encontrándolos uno con otro por sus superficies impregnadas del fluido vacuno, y ajustándolos perfectamente, se cubren de cera derretida por todos sus bordes, para impedir el contacto del aire. Cuanto más iguales y bruñidas sean las superficies, estarán más impenetrables al aire. El Dr. Dn. Juan Manuel Pérez, no satisfecho con pulimentar las superficies internas de ambos cristales, ha hecho labrar en medio al tallista, una cavidad circular donde quepa el fluido vacuno sin alterarse, para remitir el fluido vacuno a América u otras distancias semejantes».

\section{Agradecimientos}

Agradezco la inestimable ayuda del Bibliotecario de la RANM Ignacio DíazDelgado Peñas y del historiador José Luis Duro Torrijos en la transcripción y comentarios de parte del material de investigación. 